Schweizerische Zeitschrift für

Severin Bühlmann

\section{Einführung}

Getrocknete Kräuter bzw. Teile davon, wie Wurzeln, Stängel, Rinde, Blätter, Blüten, Früchte oder Samen, sind die ursprünglichste Form, in der im traditionellen China Kräutermedizin verbreitet wurde. Auch der Begriff Kräuter selbst ist zu eng gefasst: Verwendet werden auch Bäume, Sträucher, Pilze, Algen, Flechten und daneben auch tierische, mineralische und selbst Produkte menschlicher Herkunft. Nun haben diese alle bereits einen langen Entstehungsweg hinter sich, bis sie dem Patienten in verwendungsfertiger Form zur Einnahme bereitstehen. Pflanzen müssen angebaut oder wild gesammelt werden. Die qualitätsentscheidenden Faktoren sind vielfältig! Ein über viele Jahrhunderte etabliertes Know-how dient der optimalen Wirkung und der Vermeidung von unerwünschten oder toxischen Nebenwirkungen.

Die Materia Medica Chinas (Abb. 1) beschreibt rund 12000 Pflanzen, die im medizinischen Gebrauch bekannt sind. Zum Vergleich: Chinas Natur hat einen Schatz von rund 30000 Pflanzenarten, während die Schweiz bzw. Deutschland aufgrund der Eiszeiten und dem Winterfrost nur rund 4000 Arten aufweist.

Fast ein Drittel von Chinas Pflanzenwelt wird also medizinisch verwendet. Von den 12000 Mitteln kommt allerdings nur ein geringer Teil häufig und regelmässig zum Einsatz, nämlich ca. 500. Von diesen wie-

\title{
Aus der Forschung
}

Schweiz Z Ganzheitsmed 2016;28:80-84

DOI: 10.1159/000445150

\section{Rohdrogen, Granulate oder flüssige Extrakte: Was wirkt am besten? - Teil 1}

Published online: March 15, 2016 derum werden von einem Drittel regelmässig grössere Mengen umgesetzt und der Rest eher selten gebraucht.

Vergessen wird oft, dass die Qualität nicht nur von den Faktoren korrekte botanische Identität und Abwesenheit von Fremdstoffen bestimmt wird, sondern, wie das die folgende Liste und einige ausgewählte Beispiele zeigen, auch noch von vielen anderen Faktoren. Jeder kann somit, wenn qualifolg infrage stellen. Folgende Faktoren spielen eine Rolle:

- botanische Identität;

- Chargenhomogenität bzw. -varietät; tativ nicht genügend, den Therapieer-

mit dem Arzneimitts kann sich die eine oder andere Form der Verabreichung als die wirksamste anbieten. Im Folgenden werden Argumente für die verschiedenen Darreichungsformen diskutiert.

Abb. 1. Die Materia Medica Chinas.
- Klima;

- Standort (z.B. Schattenhang vs. Sonnenhang usw.);

- Wildsammlung vs. Anbau;

- Bodenbeschaffenheit;

- Wässerung und Düngung;

- Erntezeitpunkt;

- Verarbeitung: Trocknung, Schnittart, Bearbeitung (Pao Zhi-Verfahren);

- Lagerung (Feuchtigkeit, Temperatur, Schädlingsbekämpfung);

- Aufbewahrungszeit zwischen Ernte/ Bearbeitung und Verbrauch;

- Transport (Traktor, Schiffscontainer (gekühlt?), Bahn, Flugzeug);

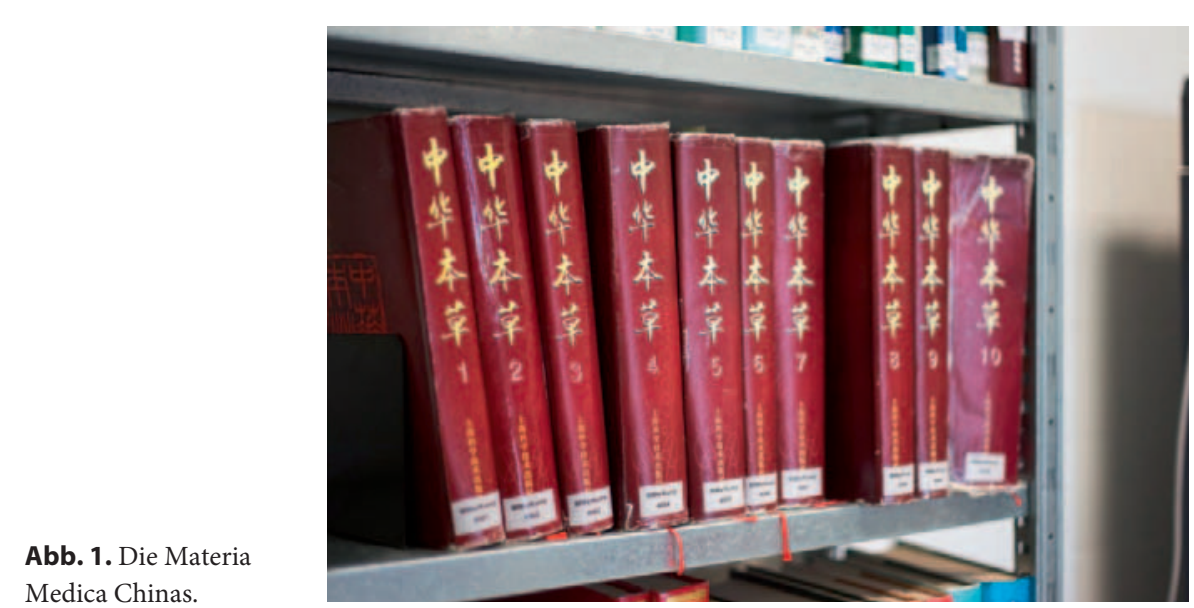

\section{KARGER}

() 2016 S. Karger GmbH, Freiburg 
- Zwischenlagerung und Zwischenbehandlung (Pestizideinsatz);

- Ausfuhr- und Einfuhrbedingungen (Gammabestrahlung);

- Spezialverarbeitung (Trockenextrakte, Sprühtrocknung, Flüssigextrakte usw.);

- Verpackungsmaterial(Licht(Stichwort: ätherische Öle) usw.);

- Endlagerung und Haltbarkeit;

- Endbehandlung (Abkochen, Aufschwemmen, Wassertemperatur, Tablettierung);

- Konsumationszeit (Magenfüllung, Magen-pH);

- individuelle Faktoren (Lebermetabolismus, Darmpassage, Interaktionen mit chemischen Medikamenten usw.);

- Fremdstoffbelastung (Pestizide, Aflatoxine, mikrobiologische Verunreinigungen usw.);

- Nachfrage und Verfügbarkeit;

- Preis;

- Dosis;

- Endzweck (Diagnosestellung, Erfahrung des Therapeuten);

- Kontinuität der Behandlung und Compliance;

- Therapeut-Patient-Beziehung (Erwartungshaltung, Placeboeffekt, Weisskitteleffekt);

- Öffentlichkeitswahrnehmung von Traditioneller Chinesischer Medizin (TCM) und China (politische Stimmung, "China-Bashing»).

Diese Auflistung ist nicht vollständig. Jeder Faktor hat einen kleineren oder grösseren Einfluss auf den Therapieerfolg, und ein Manko bei einem der vielen Punkte kann bereits den ganzen Therapieerfolg infrage stellen bzw. zunichte machen. Auf jeden einzelnen Aspekt könnte detailliert eingegangen werden.

\section{Rohdrogen}

Als Rohdrogen wird Material bezeichnet, das landläufig getrocknete Kräuter genannt wird, wohl wissend, dass es sich also auch um anderes pflanzliches Material als Kräuter han- deln kann, das mitunter auch schon auf irgendeine Art und Weise verarbeitet (geröstet, fermentiert usw.) sein kann, und dass überdies auch mineralisches, tierisches und sogar menschliches Material (z.B. verkohlte Haare) unter diesem Begriff läuft.

\section{Das klassische Dekokt}

Einige TCM-Spezialisten bevorzugen die Therapie mit solchen Rohdrogen. Es bedarf eines gewissen Aufwands, also auch einer gewissen Disziplin, diese Rohdrogen zu kochen. Wurzeln müssen oft lange Zeit gekocht werden, damit sie die wichtigen Inhaltsstoffe freigeben. Traditionell wird häufig sogar in zwei Schritten gekocht: Im ersten Schritt erfolgt die Abkochung (= Dekoktieren) nur für kurze Zeit, damit die schnell flüchtigen oder hitzeempfindlichen Inhaltsstoffe wie ätherische Öle nicht zerstört werden. Die daraus resultierende Abkochung wird beiseite gestellt, und in einem zweiten Kochprozess werden nun die weiteren Bestandteile der Rezeptur über lange Zeit, manchmal bis zu einer Stunde oder mehr, gekocht. Am Schluss werden die zwei Fraktionen dann zusammengeführt und getrunken.

Da heute kaum mehr jemand eine Gross- oder Schwiegermutter zu Hause hat, die um 4 Uhr in der Früh aufsteht und dafür sorgt, dass eine solche Medizin dann um 7 Uhr verwendungsfertig bereitsteht, ist diese Art der Therapie heute im Westen eher wenig verbreitet - oder sie wird kaum je nach den Regeln der Kunst ausgeführt. Man behilft sich mit Kompromissen, die aber den Therapieerfolg mindern können. So greift man zum Dampfkochtopf, man dekoktiert in einem Schritt, man kocht für mehrere Tage und stellt den Tee in den Kühlschrank oder man verwendet Thermosflaschen. Einige Apotheken kochen die Mittel für ihre Patienten und packen das Resultat auch sofort in sterile Beutel zu Tagesportionen ab; meistens findet dies aber auch unter Verwendung eines Dampfkochtopfs oder ähnlichen Geräten statt. Man ahnt es: Je besser man es machen möchte, umso aufwendiger ist diese Verwendungsart. Im ungünstigen Fall gehen Wirkstoffe verloren, woraufhin die klinische Wirkung geschmälert wird. Der Dekoktprozess in der ursprünglichen Form stellt sicher auch bereits einen nicht zu unterschätzenden klinisch wirksamen Faktor dar, da Zeit und Musse erforderlich sind, sich einen solchen Tee zuzubereiten. Hierbei spielen sowohl psychologische als auch handfeste chemische Faktoren eine Rolle, wenn z.B. dem Kochtopf ätherische Dämpfe entweichen und sich im Haus oder auch im «Zielobjekt» Patient verbreiten. Problematisch wird es, wenn die Nachbarn oder Mitbewohner sich über die Gerüche im Treppenhaus oder in der Wohnung beschweren und diese als Gestank bezeichnen.

Am schlechtesten wirken Rohdrogen, wenn Therapeuten, die der alten chinesischen Tradition der Kräuteranwendung verbunden sind, ihren Patienten die getrockneten Kräuter verordnen, letztere diese aber im Schrank verwahren und mangels Zeit und Motivation nur sporadisch oder gar nicht abkochen.

\section{Schonendes Kochen oder Dämpfen von empfindlichen und teuren Mitteln}

Der Vollständigkeit halber sei darauf hingewiesen, dass die Herstellung eines Dekokts als Abkochung in einem oder in zwei Schritten nicht die ausschliessliche Art ist, wie in China Heilkräuter mit Wasser extrahiert werden. Besonders wertvolle oder empfindliche Pflanzen wie z.B. Ginseng werden schonend aufbereitet, was wieder auf zwei Arten geschehen kann: Die erste Form besteht aus einem Wasserbad, d.h. in einem grossen Topf steht ein kleiner Topf, der im schwach köchelnden Wasser steht. 
Im kleinen Tontopf schwimmen die wertvollen Kräuter ebenfalls im Wasser, und zwei locker sitzende Deckel schliessen diesen kleinen Topf ab. Das Wasser im kleinen Topf kocht also unter konstanten Bedingungen, und die Inhaltsstoffe bleiben dank des Zwei-Deckel-Systems weitgehend in diesem Behältnis.

Die zweite Form der besonders schonenden Anwendung besteht darin, dass wertvolle Kräuter auf Reis gelegt werden. Der Reis wird gedämpft und die Extraktion der Kräuter erfolgt nur durch den Dampf, der aus dem Reis aufsteigt und diese durchdringt. Ein Deckel verhindert auch hier, dass die wertvollen Stoffe sich allzu sehr verflüchtigen. Ein Teil der Stoffe sickert in den gedämpften Reis und verleiht diesem nebst der medizinischen Wirkung oft auch eine angenehme Geschmacksnote.

\section{Extraktion mit Alkohol}

Es wäre falsch zu meinen, im alten China wäre nur mit Wasser extrahiert worden. Selbstverständlich kommt auch Alkohol zur Anwendung. Kräuter werden in Alkohol (Reisschnaps, Reiswein usw.) eingelegt. Dieser löst andere Inhaltsstoffe aus einer Pflanze als Wasser. Die klinische Wirkung unterscheidet sich demzufolge. Manche Pflanzen eignen sich für die alkoholische Extraktion und andere wiederum nicht. Es kann sogar schädlich sein, wenn man annimmt, es spiele keine Rolle, ob mit Alkohol oder mit Wasser extrahiert wird. In China kennt man die Unterschiede in der Wirkung dieser beiden Extraktionsformen und hat genau definiert, in welcher Situation der eine oder andere Prozess zur Anwendung kommen soll und wann beide möglich sind. Dieser Punkt sollte beachtet werden, denn dessen Missachtung kann Schaden anrichten. Es sei hier kurz auf die Kava-Kava-Produkte verwiesen, die vor einigen Jahren für grossen Aufruhr in der Welt der Kräuter- medizin sorgten und zu einem Verbot dieser Produkte führten. Im weiteren Verlauf werden eher traditionelle TCM-Mittel erwähnt. Kava-Kava sei an dieser Stelle nur stellvertretend als Pflanze, die aus Polynesien stammt, für die ganze Kräutermedizin genannt. Leider betrifft das Verbot eine durchaus wertvolle Pflanze mit hervorragenden Wirkungen, wenn sie weiter traditionell verarbeitet worden wäre.

\section{Rohdrogen gemahlen in Pulverform}

Nicht alle Mittel der TCM werden als Dekokte oder anderweitige Extrakte konsumiert. Das alte China kennt eine noch schonendere Verwendung von Rohdrogen als die bereits erwähnten Anwendungsformen. Wiederum handelt es sich auch hier um in der Regel teure Kräuter und solche, die nützliche Inhaltsstoffe aufweisen, welche durch Hitze zerstört würden. In diesem Fall werden Rohdrogen lediglich pulverisiert und entweder traditionell mit Honig zu kleinen Pillen oder zu solchen von der Grösse einer Pflaume gedreht. Letztere müssen natürlich nicht ganz geschluckt werden, sondern es wird ein Stück davon abgeschnitten. In der heutigen Zeit werden gemahlene Rohdrogen oft in Kapseln abgefüllt und können so geschluckt werden. Auch für diese Verwendungsart ist traditionelles Wissen vonnöten. Nicht jedes Kraut darf auf diese Weise verwendet werden, denn ein Kochprozess findet oft auch deshalb statt, damit toxische Bestandteile vernichtet werden oder zwischen einzelnen Kräutern einer Mixtur gewisse chemische Prozesse ablaufen, welche die klinische Wirkung beeinflussen. Das traditionelle Know-how zu dieser Verwendungsform muss vorhanden sein, wenn so vorgegangen werden soll. Moderne wissenschaftliche bzw. chemisch-pharmakologische Erkenntnisse können unter Umständen dieses Know-how ergänzen.

\section{Verwendungsfertige Extrakte: Getrocknete Extrakte}

Seit 1946 sind chinesische «Kräuter» auch als sogenannte Granulate auf dem Markt. Der Gründer Hong Yen Hsu von SunTen war der Erste, der diese Technologie in Taiwan etablierte. Er hatte sie während seiner Ausbildung zum Apotheker in Japan kennengelernt. Die Volksrepublik China wandte dieses Verfahren erst viele Jahrzehnte später an.

\section{Definition von Granulaten gemäss} der Europäischen Pharmakopöe

Bei diesem Extraktionsprozess werden einzelne Kräuter oder Mischungen davon in Anlehnung an den traditionellen Dekoktiervorgang schonend gekocht (Abb. 2). In einem nächsten Schritt wird der Suppe bzw. dem Tee das Wasser weitgehend entzogen. Es bleibt eine dickflüssige Masse, also eine Art Konzentrat, übrig. Dieses wird dann auf die Trägersubstanz Stärke aufgetragen und im Luftstrom verwirbelt, sodass schliesslich ein Pulver von gleichförmiger Korngrösse vorliegt. In dieser Form halten sich die Inhaltsstoffe über lange Zeit stabil. Das Granulat kann vom Patienten mit warmem Wasser aufgeschwemmt und so konsumiert werden. Bei der Herstellung kommen je nach Ausgangsmaterial noch einige technische Tricks zur Anwendung. Beispielsweise werden flüchtige ätherische Öle separat aufgefangen und dem Produkt kurz vor Produktionsende beim Verwirbeln mit Stärke wieder zugeführt. So werden sie weder zerstört noch gehen sie über die Luft verloren.

Granulate haben gegenüber Rohdrogen mehrere Vorteile: Als verwendungsfertige Mittel erhöhen sie die Compliance beim Patienten stark. Langzeitanwendungen sind möglich. Auch auf Reisen bewährt sich diese Form. Dank dem an die klassische Dekoktherstellung angelehnten Produktionsprozess wird eine vergleichbare Ausbeute an Wirkstoffen erzielt. 


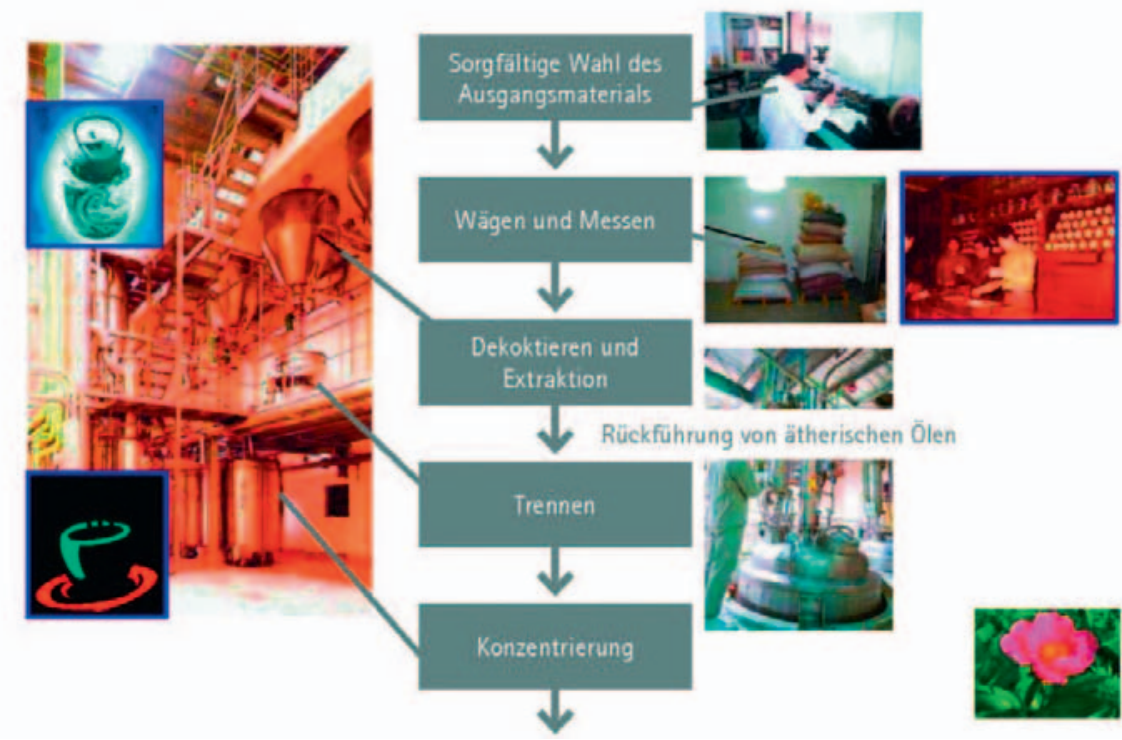

Abb. 2. Herstellungsprozess.

Durch die Konzentrierung dieser Stoffe kann bei Kenntnis des Konzentrationsfaktors die Einnahmemenge im Vergleich zum klassischen Dekokt berechnet und mengenmässig mit einer geringen Dosis gearbeitet werden. Im Vergleich zu Rohdrogen ist der Preis gemessen an klinisch vergleichbaren Mengen geringer.

\section{Flüssige Extrakte}

Der Wunsch, auch flüssige Konzentrate zur Verfügung zu haben, ist bei einem Teil der Patienten und Verschreiber vorhanden. Auf der Suche nach solchen Produkten in China oder Taiwan hört man in Fachkreisen immer wieder, dass man deren Herstellung versucht, aber dann wieder aufgegeben habe. Offensichtlich ist dieses Unterfangen schwieriger als theoretisch erwartet. Ganz unmöglich ist es jedoch nicht, wie der europäische und der US-amerikanische Markt dies beweisen. Allerdings macht es einen Unterschied, ob ein Hersteller sich auf die Erzeugung eines einzigen Produkts bzw. weniger Produkte konzentriert oder ob er eine Palette von meh- reren hundert Mitteln anbieten will, denn jedes einzelne Mittel stellt an den Hersteller individuelle Anforderungen und bedarf eines ausreichenden Know-hows. Diese Kenntnisse können nur in Jahren bzw. Jahrzehnten erworben werden. Hierzu gehört eine genaue Kenntnis der Inhaltsstoffe der einzelnen Mittel, und man sollte wissen, wie diese sich im Extraktionsverfahren verhalten. Ausserdem sollte man in der Lage sein, diese Inhaltsstoffe nachzuweisen. Wer sich diesbezüglich auskennt, weiss, dass das Erstellen einer Nachweismethode auch nur für einen einzigen Inhaltsstoff unter vielen in der Regel Monate dauert, und kann sich vorstellen, welche Schwierigkeiten mit der Palette der TCMPflanzen verbunden sind. Ein grosser Unterschied besteht zudem darin, ob jemand eine derartige Arbeit an einer Universität zu Forschungszwecken leistet oder ob sie für die industrielle Produktion geschieht. Im letzteren Fall müssen Analysemethoden in aufwendigen Testreihen validiert werden. Allein die Qualifikation eines Analysegeräts, das diese Arbeit leisten soll, ist mit einem riesigen finanziellen und zeitlichen Aufwand verbunden.
In den USA sind flüssige Extrakte aus TCM-Pflanzen erhältlich. Da aber dort TCM nicht als Medizin gilt, bestehen keine hohen Anforderungen an diese Mittel. Sie können somit auch nicht legal in Ländern wie der Schweiz verkauft werden, wo diese Produkte als Medizin gelten. Man erhält auch keine Dokumente zum Herstellungsprozess und zur Qualitätsanalyse aus den USA. Sie existieren nicht oder werden als Firmengeheimnis gehütet.

Leider sieht es bei in Europa hergestellten Flüssigextrakten ähnlich aus. Distributoren müssen in Länder ausweichen, wo diese Mittel ebenfalls nicht als Medizin gelten. Der Grosshandel ist somit in der Schweiz auch nicht möglich. Lediglich die weniger streng kontrollierte Anwendung als Ad-hoc-Rezeptur oder Magistralrezeptur ist erlaubt. Das Terrain, auf dem man sich dann bewegt, ist unsicher. Sollten auch lokale Behörden wie Kantonsapotheker etwas genauer hinsehen, könnte dies zu Auflagen führen, die grössere Anstrengungen erfordern und vielleicht sogar nicht erfüllbar sind.

Nichtsdestotrotz werden Flüssigextrakte fleissig beworben, und ihre Vorteile gegenüber anderen galenischen Formen werden betont. Dem Katalog an Argumenten, die dies unterstreichen, sollen nachstehend einige Betrachtungen folgen.

\section{Vergleich Trockenextrakte (sogenannte Granulate) versus Flüssigextrakte}

Anbieter von Flüssigextrakten betonen:

- Flüssigextrakte verfügen über einen Konzentrationsfaktor von 1:10 im Vergleich zum klassischen Dekokt, wogegen Granulate nur einen solchen von 1:5 haben und dieser sogar variieren kann. Daraus wird abgeleitet, dass die Wirksamkeit bei Flüssigextrakten hoch ist und bei Granulaten von der Dosierung abhänge. 
- Die Compliance sei mit Flüssigextrakten sehr gut, beim klassischen Dekokt jedoch eher niedrig und bei den Granulaten mittel bis sehr gut.

- Die Handhabung sei bei Flüssigextrakten mit keinem Aufwand verbunden und die Transportmöglichkeiten seien gut, wogegen dies beim klassischen Dekokt aufwen- dig sei und bei Granulaten heisses Wasser benötigt werde.

- Geschmacklich seien Flüssigprodukte süss, Dekokte zum Teil stark bitter und Granulate weniger, aber immer noch bitter.

- Flüssigextrakte und Granulate seien im Gegensatz zum klassischen Dekokt sehr stabil.
- Die Standarddosierung sei bei Granulaten im Gegensatz zu Flüssigextrakten nicht immer genügend.

- Dank Einheitspreis sind die Kosten bei Flüssigextrakten stabil, wogegen sie bei Rohdrogen und Granulaten variieren und bei letzteren zudem hoch seien. 